cases occasionaly which are not successful because of the use of these standardized procedures or because of regional pathology such as invasion of maxillary cancer. The procedure has to be done elsewhere away from Gasserion ganglion. In these instances, which are not very common, we chose the Sjöqvist procedure in five cases. In this country, the experience with this procedure is rarely found in the literature, but we feel that this procedure is still one of the useful ones in the field of pain surgery. We will report our five cases with special emphasis to the result of the operation and problem of recurrance of pain.

\title{
10. Studies on the Insensible Water Loss in the Neuro-Surgical Patients
}

\author{
Yasuhiro Hirayama, K. Ishizuka, M. Morooka, \\ S. Nomura, K. Ito and S. KiKuchI \\ Juntendo Univ. School of Medicine, Department of 2nd Surgery
}

To estimate the insensible water loss from the surface of the Neuro-surgical patients, authors have utilized a electrohygrometer by measuring the humeridity above the skin surface.

In the preliminary experiments, the water loss from the various parts of the body surface of the normal healthy individuals and its seasonal variation was estimated.

In the clinical appliance, a group of 33 cases with brain tumor showed marked difference on the insensible water loss between its value estimated on the back of the hand of each side, whereas no difference was observed by a normal control group of the healthy individuals and 60 cases with head injury. Such tendency was especially remarkable in cases of brain tumor, such as hypophysial tumor, third ventricle tumor and so on.

In the further investigation in a group of brain tumor, authors studied the relation between the bilateral asymmetry of quantity water loss on the back of the hand and the operation, and found its marked postoperative difference between both sides. 